\title{
Bootstrap Panel Granger-Causality between Government Budget and External Deficits for the EU
}

\author{
ANTÓNIO AFONSO \\ CHRISTOPHE RAULT
}

CESIFO WORKING PAPER NO. 2581

CAtegory 6: Fiscal Policy, Macroeconomics and Growth

MARCH 2009

An electronic version of the paper may be downloaded
- from the SSRN website: $\quad$ www.SSRN.com
- from the RePEc website:
- from the CESifo website: 


\title{
Bootstrap Panel Granger-Causality between Government Budget and External Deficits for the EU
}

\begin{abstract}
We investigate the existence of Granger-causality between current account and government budget balances over the period 1970-2007, for different EU and OECD country groupings. We use the panel-data approach of Kónya (2006), which is based on SUR systems and Wald tests with country specific bootstrap critical values. Our results show a causal relation from budget deficits to current account deficits for several EU countries: Bulgaria, Czech Republic, Estonia, Finland, France, Italy, Hungary, Lithuania, Poland, and Slovakia, along the lines of the so-called twin-deficit relationship. Considering the effective real exchange rate in the SUR system does not substantially alter the results.

JEL Code: C23, E62, F32, H62.

Keywords: panel causality tests, budget deficit, external imbalance, real exchange rates, EU, OECD.

\author{
António Afonso \\ European Central Bank \\ Directorate General Economics \\ Kaiserstrasse 29 \\ 60311 Frankfurt am Main \\ Germany \\ antonio.afonso@ecb.europa.eu
}

\author{
Christophe Rault \\ University of Orléans \\ France \\ chrault@hotmail.com
}

Rue de Blois-B.P. 6739

45067 Orléans Cedex 2
\end{abstract}

February 2009

The opinions expressed herein are those of the authors and do not necessarily reflect those of the European Central Bank or the Eurosystem. 


\section{Introduction}

The argument that a budget deficit leads to a current account deficit results from the fact that budget deficit increases the domestic interest rate, and this attracts foreign capital and induces an appreciation of the domestic currency, which in turn leads to an increase in the current account deficit. Such an effect will be more relevant the higher the economy's degree of openness. In practice, the existence of a relationship between the budgetary position of a country and its current account balance naturally needs to be assessed empirically. While several studies have analysed the existence of convergence (or divergence) between the trade and budgetary imbalances on a country basis, only a few studies have taken advantage of the panel econometrics framework, particularly to assess the question of our paper, the existence of Granger causality between the two imbalances.

Empirical analysis does not necessarily provide a positive correlation between the budget balance and the current account balance. Indeed, the existing evidence is rather dissimilar, notably regarding single equation analysis, in the sense that budget balance deteriorations may hardly impinge on the current account position. Overall there is some mixed evidence in favour of a twin-deficits relationship, but this is neither robust nor stable over time, which may imply that fiscal tightening may not diminish the current account deficit. For related empirical analysis see, for instance, Bernheim (1988), Chinn and Prasad (2003), Corsetti and Müller (2006), and Piersanti (2000), while Afonso and Rault (2008) provide for a non-exhaustive overview of studies on this topic.

Moreover, scarcely any evidence relates the specific issue of causality, either unidirectional or bidirectional, between the two imbalances.

Therefore, this paper contributes to the literature with a bootstrap panel analysis of causality between budget balances and external balances for the European Union and OECD countries, during the period 1970-2007. In the approach we use, we allow for cross-country correlation, without the need of pre-testing for unit roots, and such methodology is explained in section two. Section three reports the empirical analysis and section four concludes.

\section{Panel Granger causality test methodology}

The panel data approach developed by Kónya (2006) is based on the followings bivariate (here composed of current account balance, $c a$; and budget balance, $b u d$ ) or 
trivariate (here $c a$; $b u d$; and real effective exchange rate, rex) finite-order vector autoregressive models:

$$
\left\{\begin{array}{c}
c a_{i t}=\alpha_{1, i}+\sum_{j=1}^{p_{1 i}} \beta_{1, i, j} c a_{i, t-j}+\sum_{j=1}^{p_{2 i}} \gamma_{1, i, j} b u d_{i, t-j}+\varepsilon_{1, i, t} t=1, \ldots, T \quad i=1, \ldots, N \\
b u d_{i t}=\alpha_{2, i}+\sum_{j=1}^{p_{1 i}} \beta_{2, i, j} c a_{i, t-j}+\sum_{j=1}^{p_{2 i}} \gamma_{2, i, j} b u d_{i, t-j}+\varepsilon_{2, j, t} t=1, \ldots, T i=1, \ldots, N
\end{array}\right.
$$

and

$$
\left\{\begin{array}{l}
c a_{i t}=\alpha_{1, i}+\sum_{j=1}^{p_{1 i}} \beta_{1, i, j} c a_{i, t-j}+\sum_{j=1}^{p_{2 i}} \gamma_{1, i, j} b u d_{i, t-j}+\sum_{j=1}^{p_{3 i}} \eta_{1, i, j} r e x_{i, t-j}+\varepsilon_{1, i, t} t=1, \ldots, T \quad i=1, \ldots, N \\
b u d_{i t}=\alpha_{2, i}+\sum_{j=1}^{p_{1 i}} \beta_{2, i, j} c a_{i, t-j}+\sum_{j=1}^{p_{2 i}} \gamma_{2, i, j} b u d_{i, t-j}+\sum_{j=1}^{p_{3 i}} \eta_{2, i, j} r e x_{i, t-j}+\varepsilon_{2, i, t} t=1, \ldots, T \quad i=1, \ldots, N
\end{array}\right.
$$

where the index $i(i=1, \ldots, N)$ denotes the country, the index $t(t=1, \ldots, T)$ the period, $j$ the lag, and $p_{1 i}, p_{2 i}$ and $p_{3 i}$, indicate the longest lags in the system. The error terms, $\varepsilon_{1, i, t}$ and $\varepsilon_{2, i, t}$, are supposed to be white-noises (i.e. they have zero means, constant variances and are individually serially uncorrelated) and may be correlated with each other for a given country, but not across countries.

Systems (1) and (2) are estimated by the Seemingly Unrelated Regressions (SUR) procedure (since possible links may exist among individual regressions via contemporaneous correlation $^{1}$ within equations (1a) and (1b) of system (1); and within equations (2a) and (2b) of system (2)). Then Wald tests for Granger causality are performed with country specific bootstrap critical values generated by simulations.

With respect to system (1) for instance, in country i there is one-way Granger- causality running from bud to ca if in the first equation not all $\gamma_{1, i}$ are zero but in the second all $\beta_{2, i}$ are zero; there is one-way Granger-causality from ca to bud if in the first equation all $\gamma_{1, i}$ are zero but in the second not all $\beta_{2, i}$ are zero; there is two-way Granger-causality

\footnotetext{
${ }^{1}$ This assumption is very likely to be relevant for many macroeconomic time series for EU or OECD countries for which strong economic links exist.
} 
between ca to bud if neither all $\beta_{2, i}$ nor all $\gamma_{1, i}$ are zero; and there is no Granger-causality between ca to bud if all $\beta_{2, i}$ and $\gamma_{1, i}$ are zero ${ }^{2}$.

This procedure has several advantages. Firstly, it does not assume that the panel is homogeneous, so it is possible to test for Granger-causality on each individual panel member separately. However, since contemporaneous correlation is allowed across countries, it makes possible to exploit the extra information provided by the panel data setting. Secondly, this approach does not require pretesting for unit roots and cointegration (since country specific bootstrap critical values are generated), though it still requires the specification of the lag structure. This is an important feature since the unit-root and cointegration tests in general suffer from low power, and different tests often lead to contradictory outcomes. Thirdly, this panel Granger causality approach allows the researcher to detect for how many and for which members of the panel there exists one-way Granger-causality, two-way Granger-causality or no Granger-causality.

\section{Econometric investigation}

\subsection{Data}

All data for current account balances, general government budget balances and real effective exchange rates are taken from the European Commission AMECO (Annual Macro-Economic Data) database, from the IMF and from the OECD databases. ${ }^{3}$ We consider four different country panels: EU15, EU25, Cgroup21, and Cgroup26. The data cover respectively the periods from 1970 to 2007 for the EU15 countries, from 1996 to 2007 for the EU25 countries (i.e. EU27 without Cyprus and Romania, due to short time span availability), from 1970 to 2007 for the Cgroup21 (i.e. EU15 and Australia, Canada, Iceland, Japan, Norway, USA), and from 1987 to 2007 for Cgroup26 (i.e. EU15 and Australia, Canada, Iceland, Japan, Korea, Mexico, NewZealand, Norway, Switzerland, Turkey, USA). The unbalanced panels within the period 1970-2007 are used for the SUR analysis and Granger-causality testing.

\footnotetext{
${ }^{2}$ As stressed by Kónya (2006) this definition implies causality for one period ahead. Note that in the trivariate system our focus will remain on the bivariate, one-period-ahead relationship between $c a$ and $b u d$, so we will not study the possibility of causality at longer horizons, nor the possibility of two variables jointly causing the third one. In other words, rex is treated here as an auxiliary variable, and will not be directly involved in the Granger causality analysis.

${ }^{3}$ The AMECO codes are the following ones: .1.0.319.0.ublge, Net lending $(+)$ or net borrowing (-): general government, \% of GDP at market prices - excessive deficit procedure). 1.0.310.0.UBCA, Balance on current transactions with the rest of the world (National accounts), $\%$ of gross domestic product at market prices.
} 


\subsection{Empirical results}

We report in Tables 1 and 2 the results for the Granger causality tests, using a bivariate model, respectively from budget balances to current account balances, and from current account balances to budget balances. Those tables present results for the country groups EU15, EU25 and country group CGroup21, as previously defined. Tables 3 and 4 present a similar set of results for Granger causality tests regarding a trivariate model where the effective real exchange rate is also included, while the evidence on statistically significant causality is summarised in Table $5 .{ }^{4}$

Our results uncover the existence of one-way direct Granger causality from the government budget balance to the current account balance, in the bivariate model, for five EU countries: Bulgaria, Czech Republic, Finland, Lithuania, and Slovakia. Out of this set of countries only Finland is not a New Member State (NMS) of the EU. Interestingly, these results hold broadly when a trivariate specification is used, and the effective real exchange rate is considered. In this case, there is also evidence of one-way causality from the budget balances to the current account balances for some additional countries: Estonia, Hungary, Poland, France and Italy.

The fact that the majority of the countries, for which causality from the budget balance to the current account balance is found, are NMS, could be related to the existence of higher interest rates in those countries, high inflows of foreign investment and the appreciation of the respective domestic currencies. Notice that the time span used in the analysis for the NMS covers the period 1996-2007, when these economies followed a catching-up process, notably attracting foreign capital. Moreover, one can conjecture that government budgets also contributed to such process notably by raising internal demand. The evidence of causality from budget balances to the current balances for France and Italy can also be related to relevant budgetary imbalances and higher interest rates during the period used in the sample.

Regarding the existence of causality from the current account balances to the budget balances, there is statistical evidence for a different set of countries; seven from the EU (Austria, Belgium, Ireland, Spain, Czech Republic, Estonia, and Italy), and five other non-EU countries (Australia, Canada, Norway, Iceland, and Mexico). Such

\footnotetext{
${ }^{4}$ Additional results for the alternative country group CGroup26 are available in the Appendix.
} 
evidence is rather unchanged considering or not the effective real exchange rate in the SUR system.

\section{Conclusion}

We investigated the existence of Granger-causality between current account and government budget balances, with and without considering the effective real exchange rate, over the period 1970-2007, for several EU and OECD country groupings. We used the panel-data approach of Kónya (2006), which is based on SUR systems and Wald tests with country specific bootstrap critical values.

Our results support the hypothesis of a causal relation from budget deficits to current account deficits for several countries in the EU: Bulgaria, Czech Republic, Estonia, Finland, France, Italy, Hungary, Lithuania, Poland, and Slovakia, along the lines of the so-called twin-deficit relationship. On the other hand, the possibility of a reverse causality is found to be statistically significant for a somewhat different sub-set of OECD countries. Considering the effective real exchange rate in the SUR system does not substantially alter the causality results.

\section{References}

Afonso, A. and Rault, C. (2008). "Budgetary and external imbalances relationship: a panel data diagnostic", ECB Working Paper n. 933.

Bernheim, B. (1988). "Budget Deficits and the Balance of Trade", Tax Policy and the Economy, 2.

Chinn, D. and Prasad, E. (2003). "Medium-term determinants of current accounts in industrial and developing countries: an empirical exploration", Journal of International Economics, 59 (1), 47-76.

Corsetti, G. and Müller, G. (2006). "Twin Deficits: Squaring Theory, Evidence and Common Sense", Economic Policy, 21 (48), 597-638.

Kónya, L. (2006), "Exports and growth: Granger causality analysis on OECD countries with a panel data approach", Economic Modelling, 23, 978-982.

Piersanti, G. (2000). "Current Account Dynamics and Expected Future Budget Deficits: some International Evidence", Journal of International Money and Finance, 19 (2), 255-271. 
Table 1a - Granger causality tests from budget balances to current account balances for the EU15 panel (1970-2007), bivariate (CA, BUD) models

\begin{tabular}{lccccc}
\hline \multicolumn{1}{c}{ Country } & $\begin{array}{c}\text { Estimated } \\
\text { coefficient }\end{array}$ & Test Statistic & \multicolumn{3}{c}{ Bootstrap critical values } \\
\cline { 3 - 5 } & -0.1218 & 1.9729 & $1 \%$ & $5 \%$ & $10 \%$ \\
\hline Austria & -0.0960 & 9.0896 & 17.6089 & 9.9709 & 7.4661 \\
Belgium & 0.0091 & 0.0304 & 22.1543 & 14.934 & 10.314 \\
Denmark & -0.1801 & $10.614 * *$ & 20.6241 & 9.8911 & 6.8413 \\
Finland & -0.0955 & 2.8023 & 15.3098 & 10.1621 & 7.0973 \\
France & 0.0474 & 0.2793 & 18.5248 & 10.9349 & 7.6774 \\
Germany & -0.0146 & 0.0743 & 18.4403 & 10.3510 & 6.2865 \\
Greece & -0.0155 & 0.0491 & 21.1807 & 13.2739 & 9.5517 \\
Ireland & -0.0788 & 3.5063 & 22.1727 & 14.7907 & 9.0896 \\
Italy & -0.1158 & 0.5750 & 22.0144 & 12.6893 & 8.9588 \\
Luxembourg & 0.1178 & 1.6475 & 24.1254 & 14.7569 & 9.2305 \\
Netherlands & -0.2672 & 5.6111 & 19.9103 & 11.1683 & 7.1957 \\
Portugal & -0.0780 & 1.2446 & 17.8247 & 9.73810 & 6.8028 \\
Spain & -0.0450 & 1.5160 & 24.8919 & 13.1370 & 8.8215 \\
Sweden & -0.0025 & 0.0241 & 18.9911 & 9.98609 & 6.0055 \\
UK & N & 20.2869 & 11.7501 & 8.7638 \\
\hline \hline
\end{tabular}

Notes: a) $* * *, * *$ and $*$ denotes significance at the $1 \%, 5 \%$ and $10 \%$ levels, respectively.

b) $\mathrm{H}_{0}$ : BUD does not cause CA.

Table $1 \mathrm{~b}$ - Granger causality tests from budget balances to current account balances for the EU25 panel (1970-2007, 1996-2007 for NMS), bivariate (CA, BUD) models

\begin{tabular}{|c|c|c|c|c|c|}
\hline \multirow[t]{2}{*}{ Country } & \multirow{2}{*}{$\begin{array}{l}\text { Estimated } \\
\text { coefficient }\end{array}$} & \multirow[t]{2}{*}{ Test Statistic } & \multicolumn{3}{|c|}{ Bootstrap critical values } \\
\hline & & & $1 \%$ & $5 \%$ & $10 \%$ \\
\hline Austria & -0.1125 & 2.14762 & 43.7973 & 26.2007 & 16.9958 \\
\hline Belgium & -0.1102 & 16.1655 & 56.5276 & 29.8958 & 21.162 \\
\hline Bulgaria & -0.3940 & $46.9707 * *$ & 66.5806 & 31.3461 & 24.4404 \\
\hline Czech Republic & -0.2389 & $33.5267 *$ & 136.764 & 46.8060 & 27.8800 \\
\hline Denmark & 0.0049 & 0.01253 & 42.398 & 26.3917 & 20.1636 \\
\hline Estonia & -0.2775 & 5.90757 & 86.9449 & 40.5836 & 26.5668 \\
\hline Finland & -0.1856 & $13.3247^{*}$ & 57.5861 & 29.4169 & 12.4979 \\
\hline France & -0.1529 & 10.0342 & 61.6752 & 38.4433 & 26.5120 \\
\hline Germany & -0.0009 & 0.00176 & 48.2582 & 29.6834 & 20.4028 \\
\hline Greece & -0.0330 & 0.58713 & 46.8234 & 30.1429 & 20.8347 \\
\hline Hungary & -0.2083 & 6.38740 & 82.4022 & 36.2005 & 26.1898 \\
\hline Ireland & -0.0338 & 0.26381 & 66.935 & 35.1428 & 25.4057 \\
\hline Italy & -0.0896 & 6.66486 & 38.7987 & 25.4962 & 19.6885 \\
\hline Lithuania & -0.5114 & $25.7777 *$ & 99.1208 & 30.9541 & 20.0091 \\
\hline Luxembourg & -0.2839 & 5.46588 & 113.034 & 44.2571 & 28.8312 \\
\hline Latvia & 0.1188 & 0.26786 & 90.0605 & 39.9590 & 23.6354 \\
\hline Malta & 0.0358 & 0.08439 & 48.6874 & 23.8899 & 15.8910 \\
\hline Netherlands & 0.0693 & 0.79242 & 44.4382 & 26.0607 & 16.5963 \\
\hline Poland & -0.1027 & 2.24052 & 61.8482 & 26.5006 & 19.903 \\
\hline Portugal & -0.3014 & 8.28391 & 56.7452 & 32.3333 & 20.2260 \\
\hline Spain & -0.0845 & 1.85501 & 65.1751 & 37.3527 & 24.2919 \\
\hline Slovakia & 0.3128 & $51.0487 * *$ & 85.6138 & 31.5580 & 18.7401 \\
\hline Slovenia & 0.0357 & 0.13581 & 81.2513 & 36.751 & 22.6962 \\
\hline Sweden & -0.0349 & 1.28510 & 53.7627 & 26.3205 & 18.8356 \\
\hline UK & -0.0082 & 0.03551 & 51.0868 & 23.9186 & 18.2811 \\
\hline
\end{tabular}

Notes: a) ***,** and * denotes significance at the $1 \%, 5 \%$ and $10 \%$ levels, respectively.

b) $\mathrm{H}_{0}$ : BUD does not cause CA. 
Table 1c - Granger causality tests from budget balances to current account balances for the Cgroup21 panel (1970-2007), bivariate (CA, BUD) models

\begin{tabular}{lccccc}
\hline \hline \multicolumn{1}{c}{ Country } & $\begin{array}{c}\text { Estimated } \\
\text { coefficient }\end{array}$ & Test Statistic & \multicolumn{3}{c}{ Bootstrap critical values } \\
\cline { 3 - 6 } Australia & 0.0987 & 2.47781 & $1 \%$ & $5 \%$ & $10 \%$ \\
Austria & -0.1610 & 4.09952 & 35.3057 & 12.2437 & 8.03210 \\
Belgium & -0.0712 & 8.59699 & 48.5488 & 24.2883 & 11.8195 \\
Canada & 0.0543 & 2.59082 & 34.0876 & 19.4779 & 16.5793 \\
Denmark & 0.0238 & 0.40652 & 33.4390 & 19.0888 & 12.8996 \\
Finland & -0.1540 & $13.3932 *$ & 35.8901 & 20.3575 & 13.3019 \\
France & -0.1148 & 6.44631 & 41.9754 & 21.7033 & 15.3719 \\
Germany & -0.0118 & 0.02399 & 31.9677 & 17.1553 & 9.69146 \\
Greece & -0.0047 & 0.01174 & 35.8497 & 24.0044 & 17.3849 \\
Iceland & -0.0950 & 5.84384 & 31.2546 & 14.9175 & 10.3450 \\
Ireland & -0.0104 & 0.03865 & 41.8438 & 21.6514 & 15.7192 \\
Italy & 0.1649 & 0.86808 & 31.7055 & 18.2363 & 13.2669 \\
Japan & -0.0656 & 7.76730 & 40.9027 & 20.8168 & 15.8255 \\
Luxembourg & -0.1858 & 1.84990 & 41.3271 & 23.2244 & 14.4388 \\
Netherlands & 0.1363 & 2.76275 & 31.8408 & 17.2845 & 11.1476 \\
Norway & 0.0731 & 0.73817 & 42.6422 & 22.4094 & 15.6983 \\
Portugal & -0.2707 & 7.26021 & 35.1842 & 21.9296 & 13.7191 \\
Spain & -0.0832 & 1.57094 & 50.8964 & 24.5849 & 17.0351 \\
Sweden & -0.0465 & 1.82871 & 34.6576 & 17.3003 & 12.4461 \\
UK & 0.0376 & 0.67360 & 33.3215 & 19.2197 & 12.8902 \\
USA & -0.1016 & 7.62654 & 27.2683 & 14.5142 & 9.69367 \\
\hline \hline
\end{tabular}

Notes: a) $* * *, * *$ and $*$ denotes significance at the $1 \%, 5 \%$ and $10 \%$ levels, respectively.

b) $\mathrm{H}_{0}$ : BUD does not cause CA.

Table $2 \mathrm{a}$ - Granger causality tests from current account balances to budget balances for the EU15 panel (1970-2007), bivariate (CA, BUD) models

\begin{tabular}{lcllll}
\hline \hline \multirow{1}{*}{ Country } & $\begin{array}{c}\text { Estimated } \\
\text { coefficient }\end{array}$ & Test Statistic & \multicolumn{3}{c}{ Bootstrap critical values } \\
\cline { 3 - 5 } & & $1 \%$ & $5 \%$ & $10 \%$ \\
\hline Austria & 0.2210 & $13.4208^{* *}$ & 17.5712 & 10.4197 & 7.9679 \\
Belgium & 0.2871 & $13.7078^{* *}$ & 19.7167 & 13.5886 & 9.7279 \\
Denmark & 0.0881 & 1.31955 & 18.1164 & 12.3046 & 9.2414 \\
Finland & 0.0671 & 1.35295 & 27.2978 & 14.5593 & 10.271 \\
France & -0.064 & 1.39614 & 23.4105 & 12.7198 & 8.7197 \\
Germany & 0.1271 & 3.60793 & 18.5508 & 10.6659 & 8.0736 \\
Greece & 0.0294 & 0.27339 & 23.4356 & 13.4522 & 9.7064 \\
Ireland & 0.1696 & 12.7463 & 20.4963 & 14.3203 & 12.831 \\
Italy & 0.1876 & 2.84178 & 15.5296 & 9.48713 & 7.0580 \\
Luxembourg & -0.0011 & 0.00816 & 16.4516 & 8.72485 & 6.2463 \\
Netherlands & 0.0923 & 1.61837 & 19.0365 & 12.6034 & 8.6502 \\
Portugal & 0.0753 & 2.72063 & 20.4930 & 12.1438 & 8.6698 \\
Spain & -0.1470 & $13.7229 * *$ & 21.7501 & 12.8443 & 8.8471 \\
Sweden & 0.1281 & 2.84636 & 25.2514 & 12.8172 & 9.8969 \\
UK & -0.0120 & 0.01126 & 19.3643 & 12.5416 & 7.7915 \\
\hline \hline
\end{tabular}

Notes: a) $* * *, * *$ and $*$ denotes significance at the $1 \%, 5 \%$ and $10 \%$ levels, respectively.

b) $\mathrm{H}_{0}$ : CA does not cause BUD. 
Table $2 \mathrm{~b}$ - Granger causality tests from current account balances to budget balances for the EU25 panel (1970-2007, 1996-2007 for NMS), bivariate (CA, BUD) models

\begin{tabular}{|c|c|c|c|c|c|}
\hline \multirow[t]{2}{*}{ Country } & \multirow{2}{*}{$\begin{array}{l}\text { Estimated } \\
\text { coefficient }\end{array}$} & \multirow[t]{2}{*}{ Test Statistic } & \multicolumn{3}{|c|}{ Bootstrap critical values } \\
\hline & & & $1 \%$ & $5 \%$ & $10 \%$ \\
\hline Austria & 0.2477 & $27.0823 * *$ & 51.4192 & 27.0725 & 18.8457 \\
\hline Belgium & 0.3395 & $22.9939 *$ & 60.7129 & 33.9597 & 22.8654 \\
\hline Bulgaria & -0.1214 & 6.53548 & 81.7843 & 43.1901 & 29.6061 \\
\hline Czech Republic & 1.1697 & $280.073 * * *$ & 85.5306 & 50.0388 & 37.0912 \\
\hline Denmark & 0.0937 & 2.05593 & 65.1437 & 36.1363 & 24.1356 \\
\hline Estonia & -0.1068 & 10.3509 & 55.9677 & 27.9963 & 18.7136 \\
\hline Finland & 0.0805 & 2.91939 & 67.3981 & 36.3683 & 24.804 \\
\hline France & -0.0055 & 0.01425 & 68.8777 & 37.0922 & 25.8136 \\
\hline Germany & 0.1413 & 6.81759 & 53.1155 & 29.7250 & 20.3780 \\
\hline Greece & 0.0226 & 0.20797 & 61.2141 & 33.9221 & 23.2058 \\
\hline Hungary & 0.2563 & 21.7206 & 76.0673 & 40.0727 & 27.3466 \\
\hline Ireland & 0.1860 & 17.8076 & 69.9690 & 36.2288 & 24.8724 \\
\hline Italy & 0.2078 & 6.81759 & 56.3698 & 30.9851 & 21.3829 \\
\hline Lithuania & 0.1266 & 8.13473 & 58.6948 & 27.3797 & 17.9579 \\
\hline Luxembourg & 0.0464 & 1.08615 & 49.6066 & 25.7339 & 17.4109 \\
\hline Latvia & 0.0025 & 0.07720 & 52.4671 & 25.8900 & 17.0370 \\
\hline Malta & 0.1703 & 7.68076 & 73.7735 & 35.1349 & 23.2672 \\
\hline Netherlands & 0.0907 & 3.29045 & 60.7695 & 33.2827 & 22.7975 \\
\hline Poland & 0.2553 & 6.49466 & 66.5200 & 35.5261 & 23.944 \\
\hline Portugal & 0.0889 & 5.49646 & 55.3221 & 28.6983 & 19.2603 \\
\hline Spain & -0.1721 & $34.5352 * *$ & 62.9539 & 33.0983 & 22.6032 \\
\hline Slovakia & 0.0635 & 0.19904 & 80.6109 & 45.4936 & 30.8208 \\
\hline Slovenia & 0.0789 & 1.44234 & 147.694 & 71.0913 & 48.2888 \\
\hline Sweden & 0.2082 & 9.97688 & 65.2674 & 36.0752 & 24.7731 \\
\hline UK & 0.2477 & 1.00451 & 61.465 & 30.7354 & 21.4484 \\
\hline
\end{tabular}

Notes: a) $* * *, * *$ and $*$ denotes significance at the $1 \%, 5 \%$ and $10 \%$ levels, respectively.

b) $\mathrm{H}_{0}$ : CA does not cause BUD.

Table $2 \mathrm{c}$ - Granger causality tests from current account balances to for the Cgroup21 panel (1970-2007), bivariate (CA, BUD) models

\begin{tabular}{|c|c|c|c|c|c|}
\hline \multirow[t]{2}{*}{ Country } & \multirow{2}{*}{$\begin{array}{l}\text { Estimated } \\
\text { coefficient }\end{array}$} & \multirow[t]{2}{*}{ Test Statistic } & \multicolumn{3}{|c|}{ Bootstrap critical values } \\
\hline & & & $1 \%$ & $5 \%$ & $10 \%$ \\
\hline Australia & -0.2475 & $14.9762 *$ & 38.4361 & 22.9415 & 13.7611 \\
\hline Austria & 0.2222 & $19.5541 * *$ & 31.2584 & 17.2527 & 12.0923 \\
\hline Belgium & 0.3033 & 18.1000 & 40.3216 & 27.5948 & 19.7326 \\
\hline Canada & 0.2545 & $20.8322 *$ & 43.2188 & 24.4248 & 19.5489 \\
\hline Denmark & 0.0624 & 0.70461 & 41.6768 & 22.4323 & 16.6925 \\
\hline Finland & 0.0562 & 1.18696 & 42.9425 & 25.2027 & 18.3524 \\
\hline France & -0.0112 & 0.05611 & 34.2874 & 20.4964 & 15.2943 \\
\hline Germany & 0.0746 & 1.63140 & 24.0558 & 15.5602 & 10.4867 \\
\hline Greece & 0.0300 & 0.35163 & 43.7234 & 19.7262 & 14.4036 \\
\hline Iceland & 0.2236 & 7.22785 & 26.6495 & 15.3333 & 11.7551 \\
\hline Ireland & 0.1769 & $20.2250 *$ & 55.4867 & 27.1829 & 18.8700 \\
\hline Italy & -0.1497 & $33.2625 * * *$ & 30.3124 & 16.9951 & 11.8789 \\
\hline Japan & 0.1323 & 0.98644 & 37.6086 & 20.1220 & 13.3431 \\
\hline Luxembourg & 0.0352 & 0.63578 & 39.3196 & 16.8312 & 12.0791 \\
\hline Netherlands & 0.0590 & 0.87512 & 29.2102 & 16.6555 & 12.4642 \\
\hline Norway & 0.2238 & $43.9796 * *$ & 46.2584 & 23.4637 & 15.2612 \\
\hline Portugal & 0.0330 & 0.76715 & 33.6022 & 19.7013 & 12.0043 \\
\hline Spain & -0.1481 & $15.3984^{*}$ & 31.0567 & 20.1789 & 13.2209 \\
\hline Sweden & 0.1228 & 2.94902 & 42.7628 & 21.6023 & 16.4864 \\
\hline UK & -0.0863 & 0.64471 & 30.3871 & 18.1017 & 12.9953 \\
\hline USA & 0.0641 & 1.85154 & 31.0897 & 21.2929 & 15.9826 \\
\hline
\end{tabular}

Notes: a) $* * *, * *$ and $*$ denotes significance at the $1 \%, 5 \%$ and $10 \%$ levels, respectively.

b) $\mathrm{H}_{0}$ : CA does not cause BUD. 
Table 3a-Granger causality tests from budget balances to current account balances for the EU15 panel (1970-2007), trivariate (CA, BUD, REX) models

\begin{tabular}{lcllll}
\hline \hline \multicolumn{1}{c}{ Country } & $\begin{array}{c}\text { Estimated } \\
\text { coefficient }\end{array}$ & Test Statistic & \multicolumn{3}{c}{ Bootstrap critical values } \\
\cline { 3 - 6 } & -0.1026 & 0.5097 & $1 \%$ & $5 \%$ & $10 \%$ \\
\hline Austria & -0.0850 & 5.7613 & 24.4734 & 14.9480 & 7.2724 \\
Belgium & 0.0095 & 0.0330 & 18.7587 & 9.20970 & 7.0200 \\
Denmark & -0.1755 & $8.6874 *$ & 16.0793 & 8.94410 & 6.8819 \\
Finland & -0.1275 & 4.9532 & 16.1329 & 10.6111 & 8.0132 \\
France & 0.0139 & 0.0244 & 16.7405 & 10.1613 & 7.0988 \\
Germany & 0.0650 & 0.9785 & 21.2359 & 12.6377 & 8.6260 \\
Greece & -0.0333 & 0.2197 & 21.5234 & 13.5478 & 10.205 \\
Ireland & -0.1052 & 5.3060 & 14.5762 & 8.51481 & 6.7535 \\
Italy & -0.0979 & 0.2955 & 24.4612 & 11.7918 & 7.7393 \\
Luxembourg & 0.1035 & 1.2904 & 20.7780 & 9.73091 & 6.7232 \\
Netherlands & -0.2829 & 5.8426 & 15.6555 & 10.3215 & 7.8092 \\
Portugal & 0.0357 & 0.1765 & 19.3505 & 12.3120 & 8.0825 \\
Spain & -0.0628 & 2.5410 & 15.8202 & 7.81911 & 5.4434 \\
Sweden & 0.0264 & 0.2095 & 17.8724 & 9.12395 & 6.2212 \\
UK & UK
\end{tabular}

Notes: a) ***,** and * denotes significance at the $1 \%, 5 \%$ and $10 \%$ levels, respectively.

b) $\mathrm{H}_{0}$ : BUD does not cause CA.

Table $3 b$ - Granger causality tests from budget balances to current account balances for the EU25 panel (1970-2007, 1996-2007 for NMS), trivariate (CA, BUD, REX) models

\begin{tabular}{|c|c|c|c|c|c|}
\hline \multirow[t]{2}{*}{ Country } & \multirow{2}{*}{$\begin{array}{c}\text { Estimated } \\
\text { coefficient }\end{array}$} & \multirow[t]{2}{*}{ Test Statistic } & \multicolumn{3}{|c|}{ Bootstrap critical values } \\
\hline & & & $1 \%$ & $5 \%$ & $10 \%$ \\
\hline Austria & -0.0886 & 0.5542 & 41.3010 & 28.9222 & 19.2597 \\
\hline Belgium & -0.1080 & 12.467 & 52.6592 & 27.4962 & 17.2239 \\
\hline Bulgaria & -0.3604 & $30.132 *$ & 97.1317 & 46.9680 & 29.8037 \\
\hline Czech Republic & -0.2999 & $48.945 * *$ & 94.0543 & 42.4680 & 22.0298 \\
\hline Denmark & 0.0077 & 0.0312 & 41.7944 & 23.9718 & 17.2537 \\
\hline Estonia & -0.5042 & $24.019 *$ & 87.5025 & 30.0444 & 20.2046 \\
\hline Finland & -0.2016 & $14.779 *$ & 60.0785 & 30.4698 & 14.3326 \\
\hline France & -0.1610 & 11.020 & 52.2093 & 26.1209 & 18.8321 \\
\hline Germany & -0.0325 & 0.2748 & 46.2398 & 27.5085 & 18.8700 \\
\hline Greece & 0.0209 & 0.1388 & 38.2880 & 22.4442 & 16.1207 \\
\hline Hungary & -0.4590 & $25.318^{*}$ & 49.8247 & 30.8239 & 19.858 \\
\hline Ireland & -0.0525 & 0.6206 & 46.1688 & 25.7545 & 18.2065 \\
\hline Italy & -0.1011 & 6.9862 & 32.5002 & 20.6835 & 14.6002 \\
\hline Lithuania & -0.6572 & $58.804 * *$ & 233.146 & 45.1415 & 23.5948 \\
\hline Luxembourg & -0.2871 & 3.7869 & 45.0295 & 26.2708 & 19.1888 \\
\hline Latvia & -0.0694 & 0.1149 & 67.0000 & 35.8248 & 27.0371 \\
\hline Malta & -0.2203 & 0.9705 & 50.9769 & 31.7786 & 21.2535 \\
\hline Netherlands & 0.0697 & 0.7486 & 40.7907 & 23.8351 & 16.1974 \\
\hline Poland & -0.2310 & $25.698 *$ & 65.6607 & 27.4022 & 19.0187 \\
\hline Portugal & -0.2760 & 6.4198 & 34.2515 & 23.7551 & 17.9555 \\
\hline Spain & 0.0334 & 0.1867 & 22.6425 & 12.9124 & 9.4359 \\
\hline Slovakia & 0.2889 & $44.200 * *$ & 95.2704 & 31.8745 & 15.8232 \\
\hline Slovenia & 0.0376 & 0.0911 & 89.2911 & 35.7054 & 19.0756 \\
\hline Sweden & -0.0313 & 0.9367 & 54.5721 & 24.4355 & 16.4764 \\
\hline UK & -0.0011 & 0.0054 & 53.1380 & 21.5314 & 16.3642 \\
\hline
\end{tabular}

Notes: a) $* * *, * *$ and $*$ denotes significance at the $1 \%, 5 \%$ and $10 \%$ levels, respectively.

b) $\mathrm{H}_{0}$ : BUD does not cause CA. 
Table $3 \mathrm{c}$ - Granger causality tests from budget balances to current account balances for the Cgroup21 panel (1970-2007), trivariate (CA, BUD, REX) models

\begin{tabular}{lccccr}
\hline \multicolumn{1}{c}{ Country } & $\begin{array}{c}\text { Estimated } \\
\text { coefficient }\end{array}$ & Test Statistic & \multicolumn{3}{c}{ Bootstrap critical values } \\
\cline { 3 - 6 } Australia & 0.1099 & 3.1356 & $1 \%$ & $5 \%$ & $10 \%$ \\
Austria & -0.0752 & 0.3189 & 23.3271 & 13.7527 & 9.8809 \\
Belgium & -0.0713 & 6.5864 & 36.7478 & 18.3407 & 11.8442 \\
Canada & 0.0821 & 4.9609 & 37.2762 & 22.5548 & 14.6701 \\
Denmark & -0.0045 & 0.0149 & 32.2149 & 19.9396 & 13.216 \\
Finland & -0.1641 & $11.686 *$ & 29.2877 & 16.6354 & 12.2823 \\
France & -0.1647 & $12.166^{*}$ & 34.7298 & 15.2402 & 11.1207 \\
Germany & -0.0330 & 0.1901 & 27.7623 & 17.0333 & 10.8271 \\
Greece & 0.0531 & 0.8846 & 25.0917 & 12.7338 & 8.8758 \\
Iceland & -0.1230 & 8.3372 & 33.6928 & 17.3848 & 12.7044 \\
Ireland & -0.0458 & 0.6667 & 28.1537 & 13.5040 & 8.8506 \\
Italy & 0.2256 & 1.3431 & 32.9734 & 21.7877 & 15.2148 \\
Japan & -0.0430 & 2.6465 & 35.0153 & 18.2313 & 12.5074 \\
Luxembourg & -0.1024 & 0.4008 & 42.5990 & 21.2979 & 13.5377 \\
Netherlands & 0.1233 & 2.1646 & 24.8104 & 14.4957 & 11.1564 \\
Norway & 0.0683 & 0.7042 & 27.7951 & 12.5450 & 9.8458 \\
Portugal & -0.2600 & 5.7540 & 32.0350 & 17.1179 & 11.8143 \\
Spain & 0.0760 & 0.8992 & 34.4611 & 18.1117 & 11.9898 \\
Sweden & -0.0484 & 1.8375 & 15.6560 & 9.01711 & 6.8910 \\
UK & 0.0622 & 1.4544 & 32.9308 & 19.4626 & 11.2882 \\
USA & -0.0933 & 5.3514 & 28.3879 & 15.3956 & 9.7831 \\
\hline \hline
\end{tabular}

Notes: a) $* * *, * *$ and $*$ denotes significance at the $1 \%, 5 \%$ and $10 \%$ levels, respectively.

b) $\mathrm{H}_{0}$ : BUD does not cause CA.

Table $4 \mathrm{a}$ - Granger causality tests from current account balances to budget balances for the EU15 panel (1970-2007), trivariate (CA, BUD, REX) models

\begin{tabular}{|c|c|c|c|c|c|}
\hline \multirow[t]{2}{*}{ Country } & \multirow{2}{*}{$\begin{array}{l}\text { Estimated } \\
\text { coefficient }\end{array}$} & \multirow[t]{2}{*}{ Test Statistic } & \multicolumn{3}{|c|}{ Bootstrap critical values } \\
\hline & & & $1 \%$ & $5 \%$ & $10 \%$ \\
\hline Austria & 0.3105 & $23.0243 * *$ & 23.9905 & 13.1451 & 8.6418 \\
\hline Belgium & 0.3299 & $10.9965^{*}$ & 18.9138 & 13.7146 & 8.4004 \\
\hline Denmark & 0.2099 & 4.19445 & 21.0953 & 11.4226 & 8.5290 \\
\hline Finland & -0.1123 & 3.00617 & 23.3950 & 14.8938 & 10.492 \\
\hline France & -0.0328 & 0.32184 & 21.7152 & 13.9151 & 9.5126 \\
\hline Germany & 0.1021 & 1.73900 & 16.9003 & 10.2410 & 7.2934 \\
\hline Greece & 0.1403 & 6.58474 & 20.7480 & 12.2177 & 7.9267 \\
\hline Ireland & 0.1476 & $9.74008 *$ & 26.8709 & 13.1867 & 9.6735 \\
\hline Italy & 0.1957 & 2.68075 & 19.8949 & 9.27920 & 6.7191 \\
\hline Luxembourg & 0.0304 & 0.32526 & 18.5223 & 9.44810 & 6.4958 \\
\hline Netherlands & 0.1237 & 2.38242 & 17.6683 & 11.3519 & 8.0518 \\
\hline Portugal & 0.0961 & 2.81910 & 16.6935 & 11.2037 & 6.9183 \\
\hline Spain & -0.2414 & $21.1316^{* * *}$ & 19.2807 & 12.3846 & 7.0909 \\
\hline Sweden & 0.1363 & 1.16733 & 20.6931 & 12.7806 & 8.8891 \\
\hline UK & -0.0366 & 098063 & 20.4702 & 9.2977 & 6.6076 \\
\hline
\end{tabular}

Notes: a) $* * *, * *$ and $*$ denotes significance at the $1 \%, 5 \%$ and $10 \%$ levels, respectively.

b) $\mathrm{H}_{0}$ : CA does not cause BUD. 
Table $4 \mathrm{~b}$ - Granger causality tests from current account balances to budget balances for the EU25 panel (1970-2007, 1996-2007 for NMS), trivariate (CA, BUD, REX) models

\begin{tabular}{|c|c|c|c|c|c|}
\hline \multirow[t]{2}{*}{ Country } & \multirow{2}{*}{$\begin{array}{l}\text { Estimated } \\
\text { coefficient }\end{array}$} & \multirow[t]{2}{*}{ Test Statistic } & \multicolumn{3}{|c|}{ Bootstrap critical values } \\
\hline & & & $1 \%$ & $5 \%$ & $10 \%$ \\
\hline Austria & 0.3050 & $29.04767 * *$ & 42.7471 & 24.1836 & 16.5038 \\
\hline Belgium & 0.4419 & $24.14173 * *$ & 44.5815 & 22.9847 & 16.0922 \\
\hline Bulgaria & 0.1012 & 4.921740 & 83.4110 & 47.5966 & 32.8049 \\
\hline Czech Republic & 1.0058 & $189.0767 * * *$ & 81.9272 & 47.2400 & 33.9191 \\
\hline Denmark & 0.3214 & 12.06610 & 45.6130 & 23.8499 & 16.3219 \\
\hline Estonia & -0.4970 & $63.51770 * *$ & 76.1912 & 40.1880 & 26.3485 \\
\hline Finland & -0.0743 & 1.722820 & 52.7297 & 29.5373 & 19.9807 \\
\hline France & 0.0400 & 0.655909 & 56.3607 & 29.6258 & 20.3324 \\
\hline Germany & 0.0959 & 2.407945 & 48.4721 & 26.2896 & 17.8287 \\
\hline Greece & 0.1639 & 12.14297 & 40.1092 & 21.0431 & 14.6201 \\
\hline Hungary & 0.0796 & 2.062641 & 72.1796 & 33.6867 & 22.3112 \\
\hline Ireland & 0.1083 & 6.430092 & 48.7012 & 26.8627 & 18.9128 \\
\hline Italy & 0.2795 & 10.92041 & 48.7678 & 26.3480 & 17.9711 \\
\hline Lithuania & 0.1714 & 7.143020 & 76.0781 & 36.7169 & 23.5873 \\
\hline Luxembourg & 0.0540 & 1.890863 & 46.5044 & 24.769 & 15.9973 \\
\hline Latvia & -0.1244 & 14.67002 & 65.3116 & 31.6509 & 20.1739 \\
\hline Malta & 0.1228 & 4.605291 & 64.0031 & 29.6390 & 19.2660 \\
\hline Netherlands & 0.1049 & 2.800345 & 48.6511 & 25.6354 & 17.4253 \\
\hline Poland & 0.1196 & 1.117126 & 80.1080 & 41.3626 & 28.829 \\
\hline Portugal & 0.1200 & 7.354238 & 49.0942 & 25.1161 & 17.5189 \\
\hline Spain & -0.2368 & $30.73627 * *$ & 50.2643 & 27.6916 & 19.2412 \\
\hline Slovakia & -0.5469 & 10.61160 & 84.9531 & 46.0851 & 30.9879 \\
\hline Slovenia & -0.1804 & 27.24954 & 109.768 & 47.6334 & 29.6841 \\
\hline Sweden & 0.1536 & 2.100697 & 61.2883 & 32.0168 & 22.1453 \\
\hline UK & -0.1172 & 1.747915 & 49.2910 & 25.5140 & 17.4726 \\
\hline
\end{tabular}

Notes: a) ${ }^{* * *}, * *$ and $*$ denotes significance at the $1 \%, 5 \%$ and $10 \%$ levels, respectively.

b) $\mathrm{H}_{0}$ : CA does not cause BUD.

Table $4 c$ - Granger causality tests from current account balances to budget balances for the Cgroup21 panel (1970-2007), trivariate (CA, BUD, REX) models

\begin{tabular}{lllllr}
\hline \hline \multicolumn{1}{c}{ Country } & $\begin{array}{c}\text { Estimated } \\
\text { coefficient }\end{array}$ & Test Statistic & \multicolumn{3}{c}{ Bootstrap critical values } \\
\cline { 3 - 5 } Australia & -0.2085 & 8.1858 & $1 \%$ & $5 \%$ & $10 \%$ \\
Austria & 0.3374 & $45.209^{* * *}$ & 33.6979 & 16.6226 & 10.5763 \\
Belgium & 0.3755 & $17.004^{* *}$ & 30.0088 & 17.1039 & 12.9309 \\
Canada & 0.1627 & 6.5972 & 26.8670 & 14.7324 & 9.5865 \\
Denmark & 0.2481 & 7.0469 & 42.2218 & 21.7255 & 15.0578 \\
Finland & -0.0810 & 1.8025 & 35.2888 & 16.9941 & 10.532 \\
France & 0.0212 & 0.1746 & 36.4320 & 21.1240 & 15.4358 \\
Germany & 0.0324 & 0.2568 & 30.4258 & 18.2855 & 12.3405 \\
Greece & 0.1410 & 7.8410 & 26.4979 & 17.2037 & 11.9593 \\
Iceland & 0.2060 & 4.3591 & 30.2080 & 16.4601 & 11.2862 \\
Ireland & 0.1521 & $15.520^{*}$ & 24.8221 & 14.9135 & 10.6421 \\
Italy & -0.1605 & $29.502^{* * *}$ & 36.7788 & 20.5024 & 13.7037 \\
Japan & 0.1759 & 1.3964 & 29.1906 & 13.2997 & 9.52901 \\
Luxembourg & 0.0399 & 0.8494 & 28.5556 & 17.5338 & 11.8866 \\
Netherlands & 0.0868 & 1.5917 & 33.1472 & 16.4251 & 12.0154 \\
Norway & 0.1954 & $36.086^{* * *}$ & 26.0322 & 14.9633 & 11.0924 \\
Portugal & 0.0562 & 1.3120 & 35.9175 & 21.9975 & 13.9159 \\
Spain & -0.2598 & $27.506^{* *}$ & 35.1937 & 20.8754 & 12.5872 \\
Sweden & 0.0616 & 0.3297 & 33.6013 & 20.1821 & 14.6272 \\
UK & -0.1128 & 1.2055 & 35.1872 & 21.9195 & 16.0852 \\
USA & -0.0139 & 0.0740 & 27.8089 & 15.8205 & 12.1298 \\
\hline \hline Notes:a) & $* * * 17 \%$ & 27.6474 & 17.1973 & 12.1993 \\
\hline
\end{tabular}

Notes: a) $* * *, * *$ and $*$ denotes significance at the $1 \%, 5 \%$ and $10 \%$ levels, respectively.

b) $\mathrm{H}_{0}$ : CA does not cause BUD. 
Table 5 - Summary of results

a) Bivariate models (CA, BUD)

\begin{tabular}{lll}
\hline \multicolumn{1}{c}{ Panel } & \multicolumn{1}{c}{$\begin{array}{c}\text { Budget balance } \Rightarrow \text { Current } \\
\text { account balance }\end{array}$} & \multicolumn{1}{c}{$\begin{array}{c}\text { Current account balance } \\
\Rightarrow \text { Budget balance }\end{array}$} \\
\hline EU15, 1970-2007 & Finland & Austria, Belgium, Spain \\
NU25, 1970-2007; & $\begin{array}{l}\text { Bulgaria, Czech Republic, Finland, } \\
\text { Lithuania, Slovakia }\end{array}$ & $\begin{array}{l}\text { Austria, Belgium, Czech Republic, } \\
\text { Spain }\end{array}$ \\
CGroup 21, 1970-2007 & Finland & $\begin{array}{l}\text { Australia, Austria, Canada, Ireland, } \\
\text { Italy, Norway, Spain }\end{array}$ \\
$\begin{array}{l}\text { CGroup 26, 1970-2007; } \\
\text { 1987-2007 for KOR, }\end{array}$ & & $\begin{array}{l}\text { Austria, Canada, Ireland, Iceland, } \\
\text { MEX, NZ, SZ, TUR }\end{array}$ \\
\hline
\end{tabular}

b) Trivariate models (CA, BUD, REX)

\begin{tabular}{lll}
\hline \multicolumn{1}{c}{ Panel } & \multicolumn{1}{c}{$\begin{array}{c}\text { Budget balance } \Rightarrow \text { Current } \\
\text { account balance }\end{array}$} & \multicolumn{1}{c}{$\begin{array}{c}\text { Current account balance } \\
\Rightarrow \text { Budget balance }\end{array}$} \\
\hline EU15, 1970-2007 & Finland & Austria, Belgium, Ireland, Spain \\
$\begin{array}{l}\text { EU25, 1970-2007; } \\
\text { NMS, 1996-2007 }\end{array}$ & $\begin{array}{l}\text { Bulgaria, Czech Republic, Estonia, } \\
\text { Finland, Hungary, Lithuania, } \\
\text { Poland, Slovakia }\end{array}$ & $\begin{array}{l}\text { Austria, Belgium, Czech Republic, } \\
\text { Estonia, Spain }\end{array}$ \\
CGroup 21, 1970-2007 & Finland, France & $\begin{array}{l}\text { Austria, Belgium, Ireland, Italy, } \\
\text { Norway, Spain }\end{array}$ \\
$\begin{array}{lll}\text { CGroup 26, 1970-2007; } \\
\text { 1987-2007 for KOR, } \\
\text { MEX, NZ, SZ, TUR }\end{array}$ & & $\begin{array}{l}\text { Austria, Belgium, Iceland, Mexico, } \\
\text { Norway, Spain }\end{array}$ \\
\hline
\end{tabular}




\section{Appendix}

Table A1 - Granger causality tests from budget balances to current account balances for the Cgroup26 panel (1970-2007, 1987-2007 for KOR, MEX, NZ, SWZ, TUR), bivariate models (CA, BUD)

\begin{tabular}{|c|c|c|c|c|c|}
\hline \multirow[t]{2}{*}{ Country } & \multirow{2}{*}{$\begin{array}{l}\text { Estimated } \\
\text { coefficient }\end{array}$} & \multirow[t]{2}{*}{ Test Statistic } & \multicolumn{3}{|c|}{ Bootstrap critical values } \\
\hline & & & $1 \%$ & $5 \%$ & $10 \%$ \\
\hline Australia & 0.0396 & 0.5593 & 55.1075 & 24.0324 & 17.2201 \\
\hline Austria & -0.1140 & 2.3616 & 60.1008 & 34.2755 & 20.7356 \\
\hline Belgium & -0.0809 & 12.297 & 82.2568 & 53.3986 & 37.0357 \\
\hline Canada & 0.0710 & 5.7590 & 83.3562 & 43.4864 & 27.5492 \\
\hline Denmark & 0.0214 & 0.3859 & 67.2480 & 41.4165 & 29.5077 \\
\hline Finland & -0.1403 & 14.848 & 72.9308 & 43.6375 & 28.6703 \\
\hline France & -0.1224 & 8.5999 & 62.6613 & 37.1268 & 26.6512 \\
\hline Germany & 0.0002 & 0.0010 & 67.9075 & 39.5944 & 25.9632 \\
\hline Greece & 0.0069 & 0.0307 & 71.6463 & 37.1319 & 24.8612 \\
\hline Iceland & 0.1544 & 1.0854 & 73.5574 & 42.9189 & 31.0736 \\
\hline Ireland & 0.0050 & 0.0109 & 106.071 & 55.2984 & 40.8419 \\
\hline Italy & -0.1225 & 10.774 & 42.8348 & 28.4209 & 20.8929 \\
\hline Japan & -0.0715 & 11.271 & 92.8476 & 46.2855 & 33.4315 \\
\hline Korea & 0.7546 & 22.235 & 84.8402 & 46.7340 & 30.7200 \\
\hline Luxembourg & -0.1490 & 1.9725 & 107.543 & 50.5564 & 37.1407 \\
\hline Mexico & -0.0023 & 0.0028 & 84.5325 & 43.2410 & 25.2736 \\
\hline Netherlands & 0.0787 & 1.2828 & 69.1598 & 34.9397 & 23.0821 \\
\hline New Zealand & -0.0400 & 0.2651 & 53.5919 & 34.5824 & 25.0705 \\
\hline Norway & 0.1024 & 1.8895 & 75.7452 & 44.1814 & 29.8283 \\
\hline Portugal & -0.2393 & 6.0951 & 89.1114 & 29.9609 & 22.9827 \\
\hline Spain & -0.0997 & 3.4363 & 74.7362 & 41.9158 & 32.7571 \\
\hline Sweden & -0.0607 & 3.3654 & 56.6143 & 32.9059 & 24.2414 \\
\hline Switzerland & 0.0222 & 0.0313 & 76.5486 & 41.2231 & 26.9195 \\
\hline Turkey & 0.0264 & 0.2196 & 77.3461 & 36.9589 & 25.6126 \\
\hline UK & 0.0162 & 0.1479 & 70.4420 & 33.9604 & 24.3903 \\
\hline USA & -0.1024 & 10.816 & 56.0607 & 28.6441 & 24.0032 \\
\hline
\end{tabular}

Notes: a) $* * *, * *$ and $*$ denotes significance at the $1 \%, 5 \%$ and $10 \%$ levels, respectively.

b) $\mathrm{H}_{0}$ : BUD does not cause CA. 
Table A2 - Granger causality tests from current account balances to budget balances for the Cgroup26 panel (1970-2007, 1987-2007 for KOR, MEX, NZ, SWZ, TUR), bivariate models (CA, BUD)

\begin{tabular}{|c|c|c|c|c|c|}
\hline \multirow[t]{2}{*}{ Country } & \multirow{2}{*}{$\begin{array}{l}\text { Estimated } \\
\text { coefficient }\end{array}$} & \multirow[t]{2}{*}{ Test Statistic } & \multicolumn{3}{|c|}{ Bootstrap critical values } \\
\hline & & & $1 \%$ & $5 \%$ & $10 \%$ \\
\hline Australia & -0.2295 & 18.8500 & 95.5523 & 51.4872 & 33.7901 \\
\hline Austria & 0.2078 & $25.2311^{*}$ & 68.9876 & 31.1929 & 20.0492 \\
\hline Belgium & 0.3264 & 27.2304 & 68.6407 & 41.3540 & 29.6090 \\
\hline Canada & 0.2546 & $39.4861 *$ & 120.248 & 55.5246 & 42.0624 \\
\hline Denmark & 0.1170 & 3.82211 & 84.5530 & 51.3559 & 34.5897 \\
\hline Finland & 0.1194 & 8.21755 & 83.7567 & 49.5890 & 37.4456 \\
\hline France & 0.0214 & 0.26295 & 86.9724 & 56.5540 & 37.8702 \\
\hline Germany & 0.1179 & 6.32867 & 68.7082 & 41.2797 & 28.7122 \\
\hline Greece & -0.0169 & 0.13518 & 79.0020 & 45.0788 & 29.7468 \\
\hline Iceland & -0.1497 & $48.3506 * *$ & 59.1738 & 34.3845 & 19.0350 \\
\hline Ireland & 0.1672 & $29.4512 *$ & 103.256 & 51.5720 & 24.9189 \\
\hline Italy & 0.1804 & 6.04202 & 59.7671 & 32.4693 & 22.9949 \\
\hline Japan & 0.0723 & 0.42799 & 80.7655 & 43.5128 & 26.9547 \\
\hline Korea & 0.0399 & 2.73832 & 60.2762 & 37.0375 & 23.0391 \\
\hline Luxembourg & 0.0295 & 0.69214 & 64.1683 & 31.9305 & 20.7906 \\
\hline Mexico & -0.7699 & $63.1454 * *$ & 87.9046 & 45.8730 & 30.1348 \\
\hline Netherlands & 0.0144 & 0.07535 & 58.1079 & 31.6530 & 24.1077 \\
\hline New Zealand & -0.0370 & 0.07535 & 75.1239 & 40.8693 & 24.0152 \\
\hline Norway & 0.2086 & $59.3459 * *$ & 78.9056 & 45.2422 & 31.2220 \\
\hline Portugal & 0.0234 & 0.57682 & 71.8161 & 49.2144 & 27.7985 \\
\hline Spain & -0.1473 & $17.8080^{*}$ & 58.0656 & 35.7859 & 17.0820 \\
\hline Switzerland & 0.0327 & 5.93418 & 100.060 & 54.7575 & 38.7311 \\
\hline Sweden & 0.1848 & 8.01904 & 91.8010 & 50.4905 & 33.3648 \\
\hline Turkey & -0.0532 & 0.91908 & 63.1078 & 30.6742 & 22.9395 \\
\hline UK & -0.1289 & 2.54084 & 70.3820 & 43.4899 & 29.6069 \\
\hline USA & 0.0584 & 1.94511 & 78.3607 & 40.4462 & 27.5752 \\
\hline
\end{tabular}

Notes: a) $* * * * *$ and $*$ denotes significance at the $1 \%, 5 \%$ and $10 \%$ levels, respectively.

b) $\mathrm{H}_{0}$ : CA does not cause BUD. 
Table A3 - Granger causality tests from budget balances to current account balances for the Cgroup26 panel (1970-2007, 1987-2007 for KOR, MEX, NZ, SWZ, TUR), trivariate models (CA, BUD, REX)

\begin{tabular}{|c|c|c|c|c|c|}
\hline \multirow[t]{2}{*}{ Country } & \multirow{2}{*}{$\begin{array}{l}\text { Estimated } \\
\text { coefficient }\end{array}$} & \multirow[t]{2}{*}{ Test Statistic } & \multicolumn{3}{|c|}{ Bootstrap critical values } \\
\hline & & & $1 \%$ & $5 \%$ & $10 \%$ \\
\hline Australia & 0.0556 & 1.1275 & 39.9353 & 22.4305 & 15.9843 \\
\hline Austria & -0.0735 & 0.3612 & 43.2500 & 30.7355 & 19.8910 \\
\hline Belgium & -0.0659 & 6.2091 & 59.5578 & 32.6318 & 21.5883 \\
\hline Canada & 0.0966 & 8.7609 & 46.6443 & 31.1869 & 21.7066 \\
\hline Denmark & 0.0050 & 0.0230 & 45.9528 & 26.1360 & 17.6729 \\
\hline Finland & -0.1393 & 10.608 & 59.4901 & 33.9534 & 23.4073 \\
\hline France & -0.1717 & 15.860 & 50.5747 & 30.6981 & 19.8585 \\
\hline Germany & -0.0593 & 0.7399 & 47.7725 & 25.3301 & 18.4327 \\
\hline Greece & 0.0691 & 1.7506 & 42.0274 & 25.6047 & 18.2143 \\
\hline Iceland & 0.2433 & 2.1268 & 54.3961 & 29.8410 & 21.7062 \\
\hline Ireland & -0.0054 & 0.0121 & 54.9843 & 35.4853 & 25.2352 \\
\hline Italy & -0.1496 & $13.736^{*}$ & 48.0327 & 18.9687 & 13.4259 \\
\hline Japan & -0.0588 & 6.3269 & 59.8752 & 38.7450 & 27.3470 \\
\hline Korea & 0.6313 & 11.814 & 56.7566 & 37.1544 & 22.7816 \\
\hline Luxembourg & -0.0884 & 0.4841 & 64.5009 & 35.8000 & 24.8792 \\
\hline Mexico & -0.0809 & 2.8496 & 35.9138 & 23.3197 & 16.4050 \\
\hline Netherlands & 0.1226 & 2.9266 & 54.0106 & 24.9500 & 16.3664 \\
\hline New Zealand & -0.0474 & 0.3156 & 48.2675 & 24.3151 & 16.1930 \\
\hline Norway & 0.0996 & 1.8809 & 52.3565 & 32.7913 & 22.1626 \\
\hline Portugal & -0.2316 & 5.0119 & 57.250 & 27.0146 & 19.3697 \\
\hline Spain & 0.0403 & 0.3199 & 24.9863 & 17.4461 & 11.2073 \\
\hline Sweden & -0.0468 & 1.8116 & 55.1535 & 29.6352 & 18.7367 \\
\hline Switzerland & 0.0222 & 0.0862 & 67.8427 & 34.4097 & 23.3951 \\
\hline Turkey & 0.0148 & 0.0455 & 48.7912 & 32.4513 & 23.1931 \\
\hline UK & 0.0302 & 0.4363 & 43.7328 & 25.7231 & 16.3385 \\
\hline USA & -0.1095 & 10.538 & 41.9155 & 21.0433 & 14.6832 \\
\hline
\end{tabular}

Notes:`a) $* * *, * *$ and $*$ denotes significance at the $1 \%, 5 \%$ and $10 \%$ levels, respectively. b) $\mathrm{H}_{0}$ : BUD does not cause CA. 
Table A4 - Granger causality tests from current account balances to budget balances for the Cgroup26 panel (1970-2007, 1987-2007 for KOR, MEX, NZ, SWZ, TUR), trivariate models

\begin{tabular}{lcllll}
\multicolumn{5}{c}{ (CA, BUD, REX) } \\
\hline \multicolumn{1}{c}{ Country } & Estimated & Test Statistic & \multicolumn{3}{c}{ Bootstrap critical values } \\
\cline { 4 - 6 } & coefficient & & $1 \%$ & $5 \%$ & $10 \%$ \\
\hline Australia & -0.1655 & 8.7104 & 54.1639 & 33.0308 & 21.8734 \\
Austria & 0.3048 & $55.245^{* * *}$ & 55.1421 & 32.6740 & 21.5371 \\
Belgium & 0.3710 & $22.438^{*}$ & 48.8503 & 28.0876 & 20.4877 \\
Canada & 0.1211 & 6.0820 & 61.8913 & 43.0496 & 31.1421 \\
Denmark & 0.2154 & 10.419 & 57.0436 & 37.0555 & 22.8651 \\
Finland & -0.0982 & 3.9222 & 59.0244 & 39.7540 & 27.8795 \\
France & 0.0382 & 0.7961 & 73.0054 & 38.2013 & 27.4366 \\
Germany & 0.0026 & 0.0022 & 56.5285 & 27.5070 & 18.5895 \\
Greece & 0.0945 & 4.7533 & 60.4405 & 28.3771 & 17.4979 \\
Iceland & -0.1654 & $45.543^{* * *}$ & 36.6003 & 19.4342 & 14.9104 \\
Ireland & 0.1381 & 16.060 & 61.6908 & 36.4965 & 24.3072 \\
Italy & 0.1870 & 4.8632 & 42.7536 & 27.0309 & 20.5896 \\
Japan & 0.0624 & 0.2977 & 54.0851 & 30.8936 & 19.7079 \\
Korea & 0.0229 & 0.8137 & 39.1036 & 23.4657 & 16.4832 \\
Luxembourg & 0.0441 & 1.5859 & 47.2989 & 29.7339 & 18.2610 \\
Mexico & -0.7731 & $47.226^{* *}$ & 76.2906 & 35.8339 & 23.9523 \\
Netherlands & 0.0246 & 0.1776 & 53.9507 & 28.1421 & 18.8478 \\
New Zealand & -0.0393 & 0.7025 & 57.8516 & 33.4878 & 22.5199 \\
Norway & 0.1742 & $42.861^{* *}$ & 75.1211 & 34.7805 & 25.6494 \\
Portugal & 0.0288 & 0.4896 & 40.5507 & 26.7923 & 18.4358 \\
Spain & -0.2841 & $41.782^{* *}$ & 61.6380 & 30.4673 & 22.0187 \\
Sweden & 0.0431 & 0.1888 & 60.4607 & 40.2029 & 30.8044 \\
Switzerland & 0.0472 & 10.632 & 73.4669 & 38.3930 & 26.6594 \\
Turkey & 0.0134 & 0.0408 & 40.6889 & 25.3480 & 17.3184 \\
UK & -0.0918 & 1.7038 & 59.8360 & 27.7520 & 20.7588 \\
USA & -0.0013 & 0.8460 & 45.7550 & 29.4962 & 22.0389 \\
\hline \hline
\end{tabular}

Notes: a) $* * *, * *$ and $*$ denotes significance at the $1 \%, 5 \%$ and $10 \%$ levels, respectively.

b) $\mathrm{H}_{0}$ : CA does not cause BUD. 


\section{CESifo Working Paper Series}

for full list see www.cesifo-group.org/wp

(address: Poschingerstr. 5, 81679 Munich, Germany, office@cesifo.de)

2521 Geir B. Asheim and Tapan Mitra, Sustainability and Discounted Utilitarianism in Models of Economic Growth, January 2009

2522 Etienne Farvaque and Gaël Lagadec, Electoral Control when Policies are for Sale, January 2009

2523 Nicholas Barr and Peter Diamond, Reforming Pensions, January 2009

2524 Eric A. Hanushek and Ludger Woessmann, Do Better Schools Lead to More Growth? Cognitive Skills, Economic Outcomes, and Causation, January 2009

2525 Richard Arnott and Eren Inci, The Stability of Downtown Parking and Traffic Congestion, January 2009

2526 John Whalley, Jun Yu and Shunming Zhang, Trade Retaliation in a Monetary-Trade Model, January 2009

2527 Mathias Hoffmann and Thomas Nitschka, Securitization of Mortgage Debt, Asset Prices and International Risk Sharing, January 2009

2528 Steven Brakman and Harry Garretsen, Trade and Geography: Paul Krugman and the 2008 Nobel Prize in Economics, January 2009

2529 Bas Jacobs, Dirk Schindler and Hongyan Yang, Optimal Taxation of Risky Human Capital, January 2009

2530 Annette Alstadsæter and Erik Fjærli, Neutral Taxation of Shareholder Income? Corporate Responses to an Announced Dividend Tax, January 2009

2531 Bruno S. Frey and Susanne Neckermann, Academics Appreciate Awards - A New Aspect of Incentives in Research, January 2009

2532 Nannette Lindenberg and Frank Westermann, Common Trends and Common Cycles among Interest Rates of the G7-Countries, January 2009

2533 Erkki Koskela and Jan König, The Role of Profit Sharing in a Dual Labour Market with Flexible Outsourcing, January 2009

2534 Tomasz Michalak, Jacob Engwerda and Joseph Plasmans, Strategic Interactions between Fiscal and Monetary Authorities in a Multi-Country New-Keynesian Model of a Monetary Union, January 2009

2535 Michael Overesch and Johannes Rincke, What Drives Corporate Tax Rates Down? A Reassessment of Globalization, Tax Competition, and Dynamic Adjustment to Shocks, February 2009 
2536 Xenia Matschke and Anja Schöttner, Antidumping as Strategic Trade Policy Under Asymmetric Information, February 2009

2537 John Whalley, Weimin Zhou and Xiaopeng An, Chinese Experience with Global 3G Standard-Setting, February 2009

2538 Claus Thustrup Kreiner and Nicolaj Verdelin, Optimal Provision of Public Goods: A Synthesis, February 2009

2539 Jerome L. Stein, Application of Stochastic Optimal Control to Financial Market Debt Crises, February 2009

2540 Lars P. Feld and Jost H. Heckemeyer, FDI and Taxation: A Meta-Study, February 2009

2541 Philipp C. Bauer and Regina T. Riphahn, Age at School Entry and Intergenerational Educational Mobility, February 2009

2542 Thomas Eichner and Rüdiger Pethig, Carbon Leakage, the Green Paradox and Perfect Future Markets, February 2009

2543 M. Hashem Pesaran, Andreas Pick and Allan Timmermann, Variable Selection and Inference for Multi-period Forecasting Problems, February 2009

2544 Mathias Hoffmann and Iryna Shcherbakova, Consumption Risk Sharing over the Business Cycle: the Role of Small Firms' Access to Credit Markets, February 2009

2545 John Beirne, Guglielmo Maria Caporale, Marianne Schulze-Ghattas and Nicola Spagnolo, Volatility Spillovers and Contagion from Mature to Emerging Stock Markets, February 2009

2546 Ali Bayar and Bram Smeets, Economic and Political Determinants of Budget Deficits in the European Union: A Dynamic Random Coefficient Approach, February 2009

2547 Jan K. Brueckner and Anming Zhang, Airline Emission Charges: Effects on Airfares, Service Quality, and Aircraft Design, February 2009

2548 Dolores Messer and Stefan C. Wolter, Money Matters - Evidence from a Large-Scale Randomized Field Experiment with Vouchers for Adult Training, February 2009

2549 Johannes Rincke and Christian Traxler, Deterrence through Word of Mouth, February 2009

2550 Gabriella Legrenzi, Asymmetric and Non-Linear Adjustments in Local Fiscal Policy, February 2009

2551 Bruno S. Frey, David A. Savage and Benno Torgler, Surviving the Titanic Disaster: Economic, Natural and Social Determinants, February 2009

2552 Per Engström, Patrik Hesselius and Bertil Holmlund, Vacancy Referrals, Job Search, and the Duration of Unemployment: A Randomized Experiment, February 2009 
2553 Giorgio Bellettini, Carlotta Berti Ceroni and Giovanni Prarolo, Political Persistence, Connections and Economic Growth, February 2009

2554 Steinar Holden and Fredrik Wulfsberg, Wage Rigidity, Institutions, and Inflation, February 2009

2555 Alexander Haupt and Tim Krieger, The Role of Mobility in Tax and Subsidy Competition, February 2009

2556 Harald Badinger and Peter Egger, Estimation of Higher-Order Spatial Autoregressive Panel Data Error Component Models, February 2009

2557 Christian Keuschnigg, Corporate Taxation and the Welfare State, February 2009

2558 Marcel Gérard, Hubert Jayet and Sonia Paty, Tax Interactions among Belgian Municipalities: Does Language Matter?, February 2009

2559 António Afonso and Christophe Rault, Budgetary and External Imbalances Relationship: A Panel Data Diagnostic, February 2009

2560 Stefan Krasa and Mattias Polborn, Political Competition between Differentiated Candidates, February 2009

2561 Carsten Hefeker, Taxation, Corruption and the Exchange Rate Regime, February 2009

2562 Jiahua Che and Gerald Willmann, The Economics of a Multilateral Investment Agreement, February 2009

2563 Scott Alan Carson, Demographic, Residential, and Socioeconomic Effects on the Distribution of $19^{\text {th }}$ Century US White Statures, February 2009

2564 Philipp Harms, Oliver Lorz and Dieter Urban, Offshoring along the Production Chain, February 2009

2565 Patricia Apps, Ngo Van Long and Ray Rees, Optimal Piecewise Linear Income Taxation, February 2009

2566 John Whalley and Shunming Zhang, On the Arbitrariness of Consumption, February 2009

2567 Marie-Louise Leroux, Endogenous Differential Mortality, Non-Contractible Effort and Non Linear Taxation, March 2009

2568 Joanna Bęza-Bojanowska and Ronald MacDonald, The Behavioural Zloty/Euro Equilibrium Exchange Rate, March 2009

2569 Bart Cockx and Matteo Picchio, Are Short-Lived Jobs Stepping Stones to Long-Lasting Jobs?, March 2009 
2570 David Card, Jochen Kluve and Andrea Weber, Active Labor Market Policy Evaluations: A Meta-analysis, March 2009

2571 Frederick van der Ploeg and Anthony J. Venables, Harnessing Windfall Revenues: Optimal Policies for Resource-Rich Developing Economies, March 2009

2572 Ondřej Schneider, Reforming Pensions in Europe: Economic Fundamentals and Political Factors, March 2009

2573 Jo Thori Lind, Karl Ove Moene and Fredrik Willumsen, Opium for the Masses? Conflict-Induced Narcotics Production in Afghanistan, March 2009

2574 Silvia Marchesi, Laura Sabani and Axel Dreher, Agency and Communication in IMF Conditional Lending: Theory and Empirical Evidence, March 2009

2575 Carlo Altavilla and Matteo Ciccarelli, The Effects of Monetary Policy on Unemployment Dynamics under Model Uncertainty - Evidence from the US and the Euro Area, March 2009

2576 Falko Fecht, Kjell G. Nyborg and Jörg Rocholl, The Price of Liquidity: Bank Characteristics and Market Conditions, March 2009

2577 Giorgio Bellettini and Filippo Taddei, Real Estate Prices and the Importance of Bequest Taxation, March 2009

2578 Annette Bergemann and Regina T. Riphahn, Female Labor Supply and Parental Leave Benefits - The Causal Effect of Paying Higher Transfers for a Shorter Period of Time, March 2009

2579 Thomas Eichner and Rüdiger Pethig, EU-Type Carbon Emissions Trade and the Distributional Impact of Overlapping Emissions Taxes, March 2009

2580 Antonios Antypas, Guglielmo Maria Caporale, Nikolaos Kourogenis and Nikitas Pittis, Selectivity, Market Timing and the Morningstar Star-Rating System, March 2009

2581 António Afonso and Christophe Rault, Bootstrap Panel Granger-Causality between Government Budget and External Deficits for the EU, March 2009 